\title{
A Fast Method to Determine Short-circuit Capacity Based on Compensation Impact Factor
}

\author{
Haifeng $\mathrm{Li}^{1}{ }^{1, *}$, Xinfu Song ${ }^{1}$, Xinmao Xia ${ }^{1}$, Gang Liang ${ }^{1}$, Yaode Huang ${ }^{1}$ and Hongyang $\mathrm{Yu}^{2}$ \\ ${ }^{1}$ State Grid Xinjiang Electric Power Co. Ltd. Economic Research Institute, Urumqi, 830000, China \\ ${ }^{2}$ State Key Laboratory of Advanced Transmission Technology (Global Electricity Interconnection Research Institute Co. Ltd) \\ Beijing, 102209, China \\ ${ }^{*}$ Corresponding author
}

\begin{abstract}
This paper proposes a fast method to determine short-circuit capacity for an AC system, which includes a compensation capacitor, to avoid sub-synchronous resonance (SSR) risk at a system planning stage. Firstly, the compensation impact factor (CIF) is calculated for generators with different short-circuit capacities. If CIF of all generators are below the threshold value, then the short-circuit capacity is the safety one. Finally, safety short-circuit capacity group is formed and strongly suggested to the system planning due to no SSR risk threating the system. The proposed method needs simple system modeling and calculation process and little information on generator shaft. Simulation results show that the proposed method can precisely determine the safety short-circuit capacity group for the system.
\end{abstract}

Keywords—sub-synchronous resonance; short-circuit capacity; compensation impact factor

\section{INTRODUCTION}

Series compensation is a widely used technology in power system due to its advantages of enhancing power transmission capacity and transient stability in an economic way [1-3]. However, generator shaft of a thermal plant has a great opportunity to exchange sub-synchronous energy with the series compensation capacitor and then sub-synchronous resonance (SSR) occurs [4-8]. Since electric strength between AC Buses, which is decided by short-circuit capacity of an equivalent system, can change the resonance frequency of the system and damping characteristics of SSR [9-12], at the system planning stage it is critical to ensure the short-circuit capacity does not make contributions to exciting SSR

The published SSR analysis method [13-18] needs complex computation process and detail data information of generators which is not available at the system planning stage. Frequency scanning method [13-14] is a fast way to determine the system resonance frequency. But for each generator, frequency scanning method needs to build equivalent model and then scan the system. Eigenvalue [15-16] and test signal [17-18] methods require detail data information of generator and its shaft model. Besides, the aforementioned 2 methods cost too much computation resource, which is dramatically increasing when the system scale and number of generator increase. Compensation impact factor (CIF) [19] method uses an equivalent source model for generators and only needs the generator capacity and voltage level. Then the CIF for each generator can be determined. With threshold value, the SSR risk can be determined for the generators. The CIF method needs simple calculation process and little information on generator shaft. Therefore, at the planning stage CIF method can be used to determine the short-circuit capacity of each equivalent system, which results in little SSR threat for the generators.

This paper uses the CIF method to swiftly and conveniently determine safety short-circuit capacity group, by which the generators are not under SSR threat. Firstly, CIF is calculated for each generator with different short-circuit capacities of equivalent systems. Then, when the CIFs of all generators are below the threshold value, the short-circuit capacity of each equivalent system is the safety short-circuit capacity. Finally, safety short-circuit capacity group is formed by each safety short-circuit capacity and adopted at the system planning stage to ensure that there is no SSR risk for the generators. The proposed method has two advantages, i.e., 1) simple calculation because of the source model for generator and calculation of CIF, and 2) little information on generator shaft which is not available at the system planning stage.

The remains of this paper are organized as follows, Section II briefly introduces the theory and calculation of CIF. Section III gives the method to obtain safety short-circuit capacity and then forms the group of safety short-circuit capacity. Section IV tests the correctness of the group and Section V draws conclusions of this paper.

\section{THE COMPENSATION IMPACT FACTOR}

CIF [19] performs the evaluation of the SSR risk caused by fix compensation capacitor for thermal generators. Little information on generator shafts are required to obtain the CIF. As a result, the equivalent model to compute CIF for generators is convenient to build, considering using power source model for the generators. Figure 1 shows active power, which are divided into "causing disturbance group" and "restraining disturbance group", for computing CIF for a generator.

In Figure 1, the generator connects to the system through a compensated line and $N$ non-compensated lines. The "noncompensated line" represents the parallel path to the compensated line. $P_{C}$ is the active power flow in the compensated line while $P_{U C-1}$ through $P_{U C-N}$ represents the active power flow going through each non-compensated line 
respectively. The positive directions of these active power flows are shown in Figure 1.

The definition of CIF is shown in (1). Assuming that a fault happens at $t_{1}$ and then the system recovers to the steady state at $t_{2}$, if the active power of the causing disturbance group line can be offset perfectly by those of the restraining disturbance group line, then the sub-synchronous disturbance energy has no path to reach the generator end. Hence, there is no interaction energy exchanged between the generator shaft and compensation capacitor, and the generator will not be under any SSR threat.

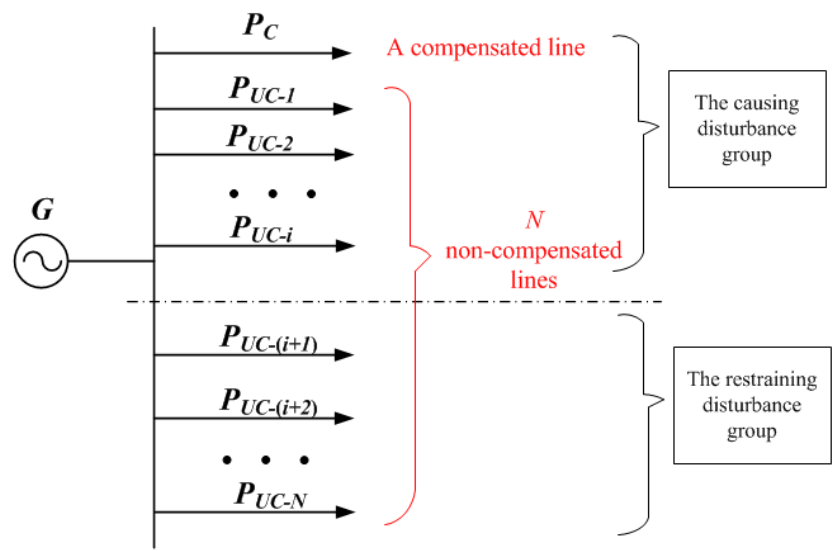

FIGURE I. ACTIVE POWER FOR COMPUTING CIF

$C I F=\left|\frac{\left[\max _{t \in\left[t_{1}, t_{2}\right]} P_{C}(\mathrm{t})-P_{C}\left(\mathrm{t}_{1}\right)\right]+\sum_{j=1}^{i}\left[\max _{t \in\left[t_{1}, t_{2}\right]} P_{U C-j}(\mathrm{t})-P_{U C-j}\left(\mathrm{t}_{1}\right)\right]}{\sum_{k=i+1}^{N}\left[P_{U C-k}\left(\mathrm{t}_{1}\right)-\min _{t \in\left[t_{1}, t_{2}\right]} P_{U C-k}(\mathrm{t})\right]}-1\right|$

However, in practical scenario, CIF is not always equal to 0 But it does not mean that SSR will occur. Therefore, [19] gives a threshold value $T_{C I F}=0.2$ to forecast that there is no SSR threat for the generator if $\mathrm{CIF}<T_{C I F}$.

\section{THE SAFETY SHORT-CIRCUIT CAPACITY}

\section{A. Equivalent Reactance}

In Figure 1 , the generator is connected to $N+1 \mathrm{AC}$ systems through the compensated line and $N$ uncompensated lines. The equivalent reactance between the generator and the $\mathrm{AC}$ system and among the AC systems can simulate short-circuit capacity of each AC system. Assuming that $X_{G-S i}$ and $X_{S i-j}$ are equivalent reactance between the generator and the $i$ th $\mathrm{AC}$ system and between the $\mathrm{AC}$ system $i$ and $j(i, j=1,2,3 \ldots N, i \neq j)$, an $\mathrm{AC}$

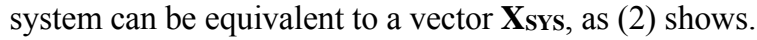

$$
X_{S Y S}(i, j, k)=\left[X_{G-S i}^{k}, \quad X_{S i-j}\right]^{T}
$$

where $k=1,2,3 \ldots m$, and $m$ is the number of generators.
With each $\mathbf{X}_{\mathbf{S Y s}}$, CIFs can be obtained for the generators and the risk of SSR can be determined. If all generators are not suffer SSR risk, then the Xsys are the safety reactance, which represents the short-circuit capacities of the equivalent $\mathrm{AC}$ systems.

\section{B. Process to Obtain the Safety Reactance}

Suppose the elements of $\mathbf{X}_{\mathbf{s y s}}$ can form $W$ different combinations which is $W \mathbf{X}_{\text {sys }}$ vectors. Figure 2 shows the process to obtain safety reactance $\mathbf{X}_{\text {sys-sty. }}$

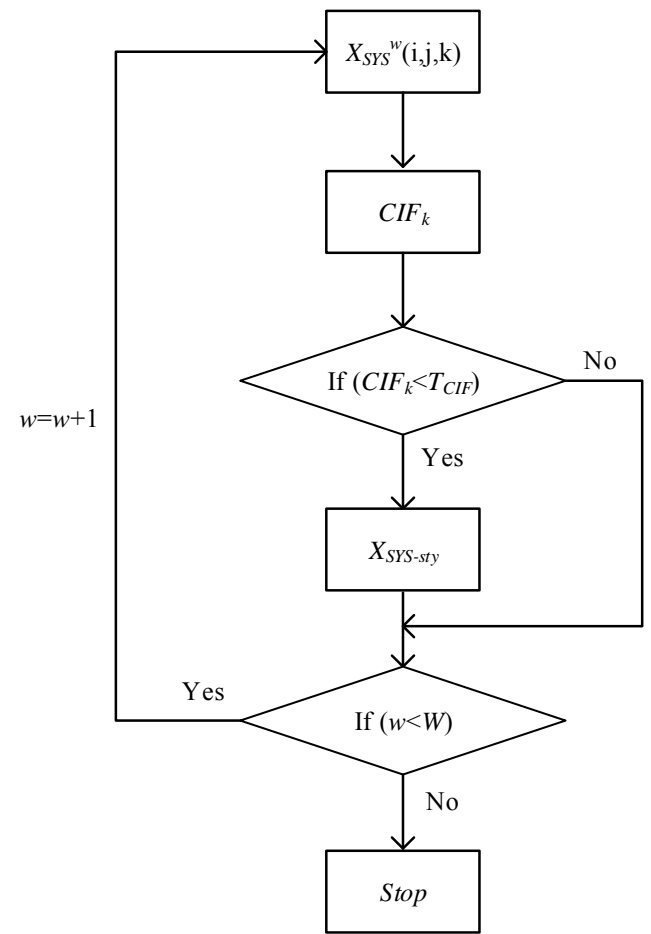

FIGURE II. THE PROCESS TO OBTAIN SAFETY REACTANCE

The process of Figure 2 to obtain $\mathbf{X}_{\mathbf{S Y S}-\text { sty }}$ can be described as follows.

a) select $w$ th combination of $\mathbf{X}_{\mathbf{S Y S}}$;

b) calculate CIF for each generator

c) if all CIFs are below the threshold value $T_{C I F}$, then the $\mathbf{X}_{\mathbf{S Y s}}$ in a) is the safety reactance $\mathbf{X}_{\mathbf{S Y s} \text {-sty; }}$

d) if $w$ is smaller than $W$, then $w=w+1$ and jump to a); otherwise stop the program.

Inverse the elements of $\mathbf{X}_{\text {sYs-sty }}$ generates the safety shortcircuit capacities of the AC systems. Then the safety shortcircuit capacity group can be formed by the safety short-circuit capacities.

\section{Advantages}

Firstly, the proposed method does not cost too much computation resource. The equivalent system model is easy to obtain and the calculation process of CIF is simple. Therefore, compared with other SSR analysis method [13-18], the proposed method is more convenient to use. 
Secondly, the proposed method can be used at system planning stage. (1) shows that the calculation of CIF requires little information on generator shaft. CIF indicates if there is a path for sub-synchronous disturbance energy to excite SSR. Therefore, without the detail data of the generator mode, CIF can be adopted to obtain safety short-circuit capacities of the AC systems.

\section{Simulation Tests}

\section{A. Test System}

Figure III shows a 3-Bus AC system with a series compensation capacitor line as the test system of this paper. $G$ and $\mathrm{T}$ represent a generator and its boost transformer respectively. $S_{1}$ and $S_{2}$ are two equivalent AC systems which are connected to Bus(2) and Bus(3) with its equivalent reactance $X_{\text {eq1 }}$ and $X_{\text {eq2 }}$. The reactance $X_{t r}$ represents the electrical strength between Bus (2) and Bus (3). $P_{t r}$ is the active power flowing through $X_{t r} . R_{1}, X_{L 1}$ and $X_{C}$ are the resistance, inductive reactance and capacitive reactance of the compensated transmission line, respectively. $R_{2}$ and $X_{L 2}$ represent the resistance and reactance of the non-compensated line. $P_{1}$ and $P_{2}$ are the active power flowing through the compensated and non-compensated transmission lines respectively. $X_{\text {eq1 }}$ and $X_{\text {eq2 }}$ belongs to $X_{G-S i}$ and $X_{t r}$ is of $X_{S i-j}$, as (2) shown. The fault at Bus (2) adopts 3-phase to ground fault to excite sub-synchronous disturbance component.

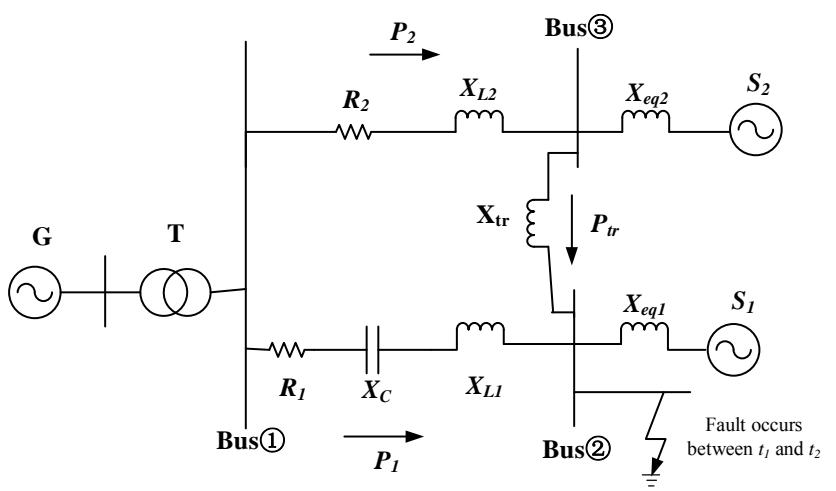

FIGURE III. THE STANDARD TEST SYSTEM

The parameters of the test system are listed in Table 1. ( $X_{\text {eq1 }}$, $\left.X_{\text {eq2 }}, X_{t r}\right)$ forms $\boldsymbol{X}_{S Y s}$ in (2). When $\left(X_{e q 1}, X_{e q 2}, X_{t r}\right)$ changes as Table 2 listed, $\boldsymbol{X}^{W_{\text {SYS }}}$ of Figure 3 is set to start the proposed method. The step of $\left(X_{e q 1}, X_{e q 2}, X_{t r}\right)$ in Table 2 is 0.01 p.u..

TABLE I. PARAMETERS OF THE EQUIVALENT SYSTEM

\begin{tabular}{|c|c|c|c|c|c|c|c|}
\hline $\begin{array}{c}\mathrm{S}_{\mathrm{B}} \\
(\mathrm{MVA})\end{array}$ & $\begin{array}{c}T \\
(\mathrm{kV})\end{array}$ & $\begin{array}{c}X_{T} \\
\text { p.u. })\end{array}$ & $\begin{array}{c}R_{1} \\
(\mathrm{pu})\end{array}$ & $\begin{array}{c}R_{2} \\
(\mathrm{pu})\end{array}$ & $\begin{array}{c}X_{L 1} \\
(\mathrm{pu})\end{array}$ & $\begin{array}{c}X_{L 2} \\
(\mathrm{pu})\end{array}$ & $\begin{array}{c}X_{C} \\
(\mathrm{pu})\end{array}$ \\
\hline 892.4 & $26 / 539$ & 0.14 & 0.02 & 0.005 & 0.417 & 0.213 & 0.204 \\
\hline
\end{tabular}

TABLE II. THE CHANGING RANGE OF THE $X_{E Q 1}, X_{E Q 2} A N D X_{T R}$

\begin{tabular}{|c|c|c|c|}
\hline Parameter & $X_{\text {eqq }}(\mathrm{pu})$ & $X_{\text {eq2 }}(\mathrm{pu})$ & $X_{\text {tr }}(\mathrm{pu})$ \\
\hline Range & $0 \sim 0.5$ & $0 \sim 0.5$ & $0 \sim 1$ \\
\hline
\end{tabular}

Note: $S_{B}=892.4 \mathrm{MVA}, V_{B}=539 \mathrm{kV}$.

\section{B. Safety Reactance}

This section calculates CIF with each point of $\left(X_{e q 1}, X_{\text {eq2 } 2}, X_{t r}\right)$ within Table 2. When CIF is smaller than $T_{C I F}=0.2$ [19], the corresponding $\left(X_{e q 1}, X_{e q 2}, X_{t r}\right)$ point is of the safety reactance Xsys-sty of Figure 2.

Figure 4 shows that the CIF with different points of $\left(X_{e q 1}\right.$, $\left.X_{\text {eq2 }}, X_{t r}\right)$. The color bar of each figure shows the value of CIF. The $\left(X_{\text {eq } 1}, X_{\text {eq2 }}, X_{t r}\right)$ in blue area belongs to $X_{\text {sys-sty. Figure } 4}$ only gives some fragment surface of the calculation result for convenient observation. The other fragment surfaces show the similar phenomenon and do not presented in this paper.

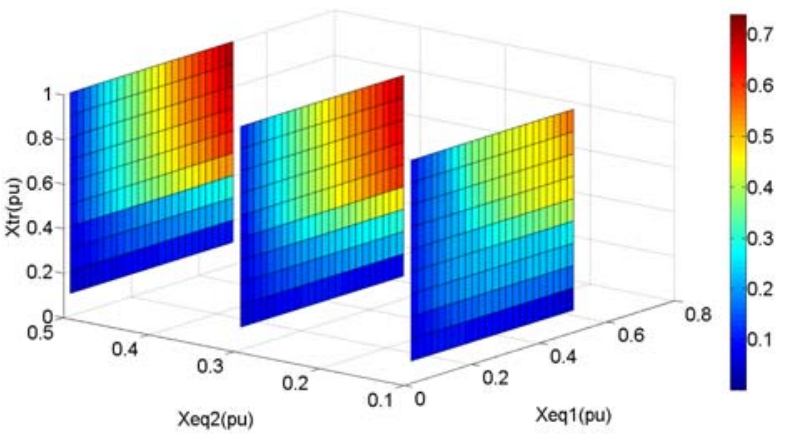

(a) The fragment surface of CIF when $X_{\text {eq }}=0.1,0.3,0.5$ p.u.

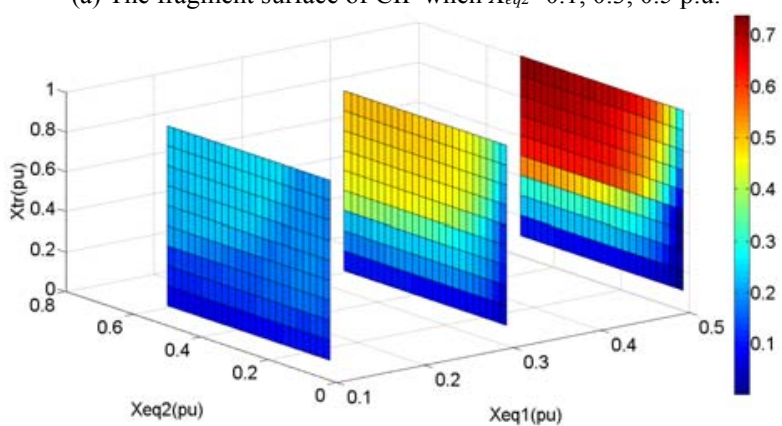

(b) The fragment surface of CIF when $X_{\text {eq1 }}=0.1,0.3,0.5$ p.u.

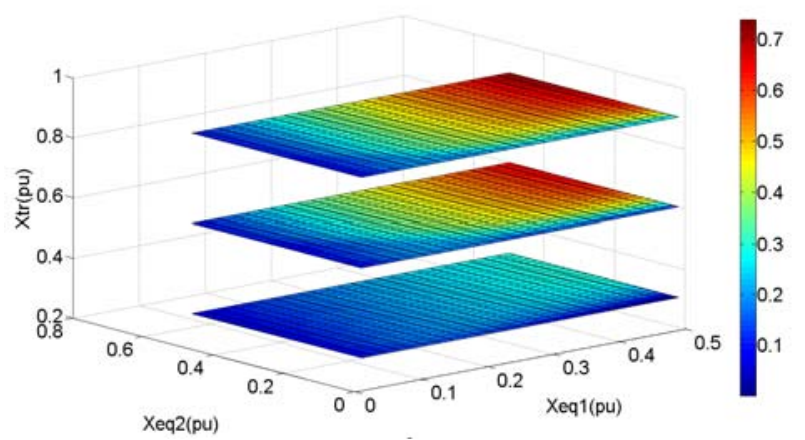

(c) The fragment surface of CIF when $X_{t r}=0.2,0.6,0.8$ p.u. FIGURE IV. THE CIF WITH $X^{W_{S Y S}}$

Figure 4(a) shows that when $X_{e q 2}$ is fixed, lower values of $X_{\text {eq1 }}$ and $X_{t r}$ result in a lower CIF value for the generator. Therefore, small reactance between Bus (1) and Bus (2) and between Bus(2) and Bus(3) assist to avoid SSR risk. When $X_{\text {eq2 }}$ becomes larger, the area of $\mathbf{X}_{\text {SYs-sty }}$ reduces.

Figure 4(b) shows that when $X_{e q 1}$ is fixed, lower values of $X_{\text {eq2 }}$ and $X_{t r}$ obtain a lower CIF value for the generator. 
Therefore, small reactance between Bus (1) and Bus (3) and between Bus (2) and Bus(3) are helpful to reduce SSR risk. Compared FIGURE IV(a) with FIGURE IV(b), the area of $\mathrm{X}_{\text {SYs-sty }}$ is more sensitive with the change of $X_{\text {eq1 } 1}$ rather than the change of $X_{\text {eq2 }}$.

Figure 4(c) shows that when $X_{\text {tr }}$ is fixed, lower values of $X_{e q 1}$ and $X_{e q 2}$ produce a lower CIF value for the generator. Therefore, small reactance between Bus (1) and Bus (2) and between Bus(1) and Bus(3) can eliminate the risk of SSR. When $X_{\text {tr }}$ increase from 0.2 to 0.6 p.u., the area of $\mathbf{X}_{\text {sys-sty }}$ reduces significantly. However, when $X_{t r}$ continuously increasing, the area of $\mathbf{X}_{\text {sYs-sty }}$ is not changing obviously.

Therefore, higher short-circuit capacities between the generator and the equivalent $\mathrm{AC}$ system and among the equivalent $\mathrm{AC}$ systems make contributions to eliminate the risk of SSR.

\section{SSR Test}

This section selects $3\left(X_{e q 1}, X_{e q 2}, X_{t r}\right)$ points to test the correctness of the safety reactance area obtained in section B. Table 3 shows that the CIF values of the $3\left(X_{\text {eq1 } 1}, X_{\text {eq2 } 2}, X_{\text {tr }}\right)$ points are smaller, near and greater than the threshold value $T_{C I F}=0.2$. which are located at Zone 1 , Zone 2 and Zone 3 respectively. Frequency spectrum analysis, damping characteristics and shaft torque are presented to prove that strong electric strength is critical for the generator to avoid the risk of SSR.

TABLE III.

PARAMETERS OF THE REACTANCE

\begin{tabular}{ccccc}
\hline \hline Case & $X_{\text {eq1 }}(\mathrm{pu})$ & $X_{\text {eq2 }}(\mathrm{pu})$ & $X_{\text {tr }}(\mathrm{pu})$ & $C I F$ \\
\hline 1 & 0.03 & 0.01 & 0.02 & 0.11 \\
2 & 0.30 & 0.16 & 0.10 & 0.23 \\
3 & 0.06 & 1.10 & 1.20 & 2.12 \\
\hline
\end{tabular}

Figure 5 shows SSR test results with the $3\left(X_{\text {eq1 } 1}, X_{\text {eq2 }}, X_{t r}\right)$ points.

Figure 5(a) shows the magnitudes of terminal voltage at sub-synchronous frequency component. In Case 1 there is no sub-synchronous disturbance at the terminal voltage while Case 2 and Case 3 have at disturbance energy at $26.5 \mathrm{~Hz}$.

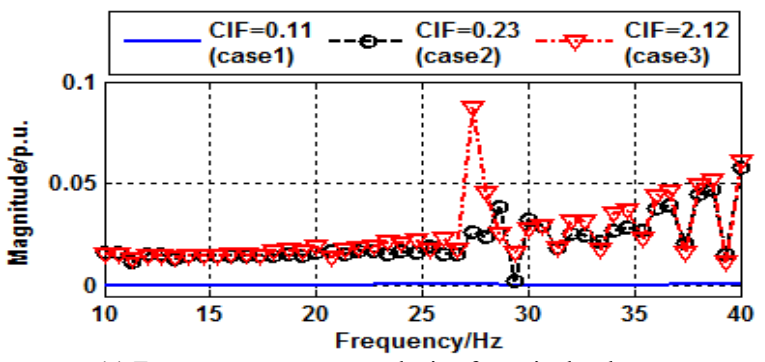

(a) Frequency spectrum analysis of terminal voltage

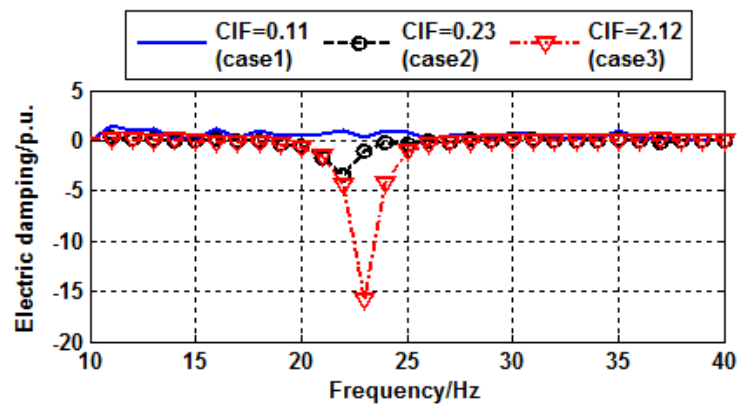

(b) Damping characteristics
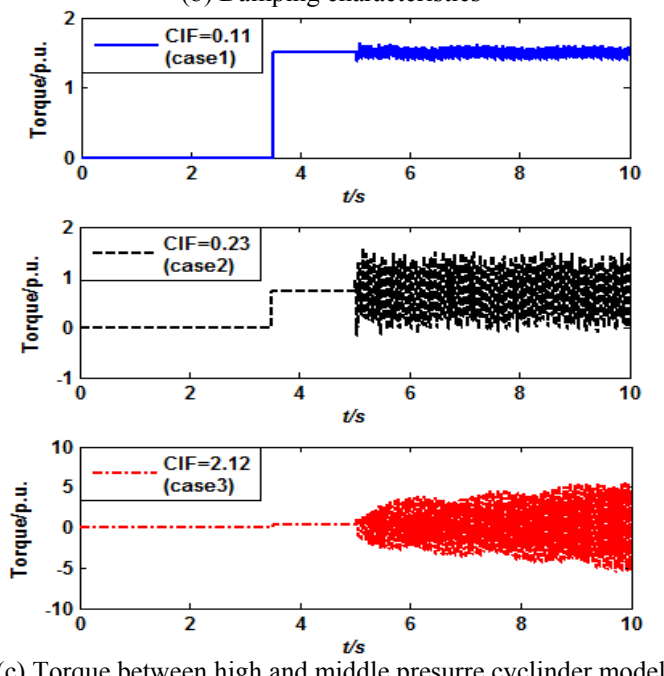

(c) Torque between high and middle presurre cyclinder model FIGURE V. SIMULATION TESTS OF SSR

Figure 5(b) shows the damping characteristic of each case. At $23.5 \mathrm{~Hz}$, the damping of Case 1 is positive while that of the other cases are negative. When CIF is near its threshold value in case 2, the damping is near 0 and the rate of oscillation divergent process are small.

Figure 5(c) are the time domain simulation of the SSR for the 3 cases. There is no SSR in case 1 because the CIF indicates that the $\left(X_{e q 1}, X_{e q 2}, X_{t r}\right)$ point is of $\mathbf{X}_{\text {SYs-sty. }}$ Case 2 and 3 show the oscillation phenomenon, especially in case 3 the oscillation is very dramatic.

Therefore, the proposed method can determine the safety reactance $\mathbf{X}_{\text {SYS-sty }}$ and help the system avoid SSR risk.

\section{CONCLUSIONS}

This paper proposes a method to obtain safety short-circuit capacity group for equivalent $\mathrm{AC}$ systems at the system planning stage. CIF is adopted to analysis the risk of SSR instead of analysis of damping characteristics. The equivalent model for CIF and its calculation process are very simple and convenient to achieve. If the CIF of $\boldsymbol{X}^{W}$ sYs is smaller than the threshold value, then $\boldsymbol{X}^{W}$ SYS is of the safety reactance $\mathbf{X}_{\text {SYS-sty }}$ which should be considered for the planning system due to no SSR risk threating the system.

Smaller reactance among the equivalent $\mathrm{AC}$ system (higher short-circuit capacity of the equivalent $\mathrm{AC}$ system) results in lager area of $\mathbf{X}_{\text {sys-sty }}$ and the sub-synchronous disturbance 
energy has no path to be transferred to the generator. Therefore, at the planning stage, smaller reactance of the equivalent system is strongly suggested to avoid the SSR risk.

\section{ACKNOWLEDGMENT}

This paper is supported by the Science and Technology project of State Grid Company of China "Research on key technology of series subsynchronous oscillation suppressor" (GEIRI-DL-71-18-005).

\section{REFERENCES}

[1] D.J. Kim, and et al, "A practical approach to HVDC system control for damping subsynchronous oscillation using the novel eigenvalue analysis program", IEEE Transactions on Power systems, vol. 22, no. 4, pp. 1926-1934, 2007.

[2] Subsynchronous Resonance Working Group of the System Dynamic Performance Subcommittee, "Reader's guide to subsynchronousresonance,"IEEE Trans. PowerSyst., vol. 7, no.1, pp. 150-157, 1992.

[3] IEEE SSR Working Group, "Terms, definitions and symbols for subsynchronousoscillation,"IEEE Trans. Power App. Syst., vol. PAS104, no.6, pp.1326-1334, 1985

[4] D.N.Walker and et al, "Resultsof subsynchronoustest at Mohave[J],'IEEE Trans. Power App.Syst., vol. PAS-94, no. 5, pp. 1878 1886, 1975

[5] H.P., Sun and et al, "Improved dynamic phasor model of HVDC system for subsynchronous oscillation study", International Conference on Electric Utility Deregulation and Restructuring and Power Technologies (DRPT), Weihai, China, pp. 485-489, 2011,

[6] N. Prabhu and et al, "Investigation of subsynchronous resonance with VSC-based HVDC transmission system,"IEEE Trans. Power Del., vol. 24, no. 1, pp. 433-440, 2009.

[7] K.R.Padiyar and et al, "Design and performance evaluation of subsynchronous damping controller with STATCOM," IEEE Trans. Power Del., vol.21, no.3, pp. 1398-1406, 2006.

[8] M.Bongiorno and et al, "A novel control strategy for subsynchronous resonance mitigation using SSSC," IEEE Trans. Power Del., vol. 23, no. 2, pp. 1033-1041, 2008.

[9] C. M. and et al, "Votage stability indices comparison on the IEEE-39 bus system using RTDS", IEEE International Conference on Power System Technology (POWERCON), Auckland, New Zealand, pp. 1-6, 2012

[10] D,Rai and et al.,"Damping inter-area oscillations using phase imbalanced series compensation schemes,"IEEE Trans. Power Syst., vol. 26, no. 3, pp. 1753-1761, 2011

[11] L. L. Fan and et al, "Modeling of DFIG-Based wind farms for SSR analysis," IEEE Trans. Power Del., vol.25, no.4, pp.2073-2083, 2010.

[12] D.Rai and et al.,"Enhancement of power system dynamics using a phase Canay1M, "A novel approach to the torsional interaction and electrical damping of the synchronous machine Part I:Theory,Part II:Application to An Arbitrary Network,'IEEE Trans. Power App. and Syst., PAS-101, no. 10, pp. $3630-3647,1982$.

[13] B.L.Agrawal and et al, "Use of frequency scanning techniques for subsynchronous resonance analysis,"IEEE Trans. Power App. Syst., vol. 98, no. 2, pp. 341-349, 1979.

[14] N.Johansson and et al, "A comparison of different frequency scanning methods for study of subsynchronous resonance,'IEEE Trans. Power Syst., vol. 26, no. 1, pp. 356-363, 2011.

[15] Yaonan Yu, "Power system dynamics," Lecture Notes. University of British Columbia, Vacouer, B.C., Canada, pp. 1970-1976.

[16] P. Kundur, Power system stability and control, New York: McGraw Hill pp. 1025-1072, 1994

[17] I. M. Canay, "A novel approach to the torsional interaction and electrical damping of the synchronous machine part I: Theory", IEEE Trans. Power App. Syst., vol. PAS-101, no. 10, pp. 3630-3638, 1982.
[18] I. M. Canay, "A novel approach to the torsional interaction and electrical damping of the synchronous machine part II: Application to an arbitrary network", IEEE Trans. Power App. Syst., vol. PAS-101, no. 10, pp. 3639-3647, 1982.

[19] H. F. Li and et al., "Screening technique for identifying the risk of SSR", IET G.T.\&D., vol. 10, no. 7, pp. 1589-1596, 2016. 